\title{
Comparison of On-line Partial Discharge Detection Methods For HV Cable Joints
}

\author{
Y. Tian, P. L. Lewin, A. E. Davies \\ Department of Electronics and Computer Science \\ University of Southampton \\ Southampton, Hampshire SO17 1BJ, UK \\ S. G. Swingler, S. J. Sutton and G. M. Hathaway \\ National Grid \\ Leatherhead, Surrey KT22 7ST, UK
}

\begin{abstract}
The capacitive coupler, acoustic emission (AE) sensor and radio frequency current transducer (RFCT) have been used to detect partial discharge (PD) activity within a $132 \mathrm{kV}$ prefabricated cable joint containing a known defect. Although each of the three methods has been applied individually under different situations, a comprehensive investigation and comparison of these three methods has not yet been made. Results obtained were compared to measurements made using the conventional electrical detection method. The quantification of the capacitive coupler measured signal in $\mathrm{mV}$ with a discharge apparent quantity in $\mathrm{pC}$ has been investigated and an on-line quantification method proposed. The capacitive coupler has good detection sensitivity and PD location can be realised by studying the time of flight between signals from two sensors. The difference in pulse shape, spectrum and time of flight between an internal discharge and external interference has been investigated. The AE approach has the advantage of being free from electrical interference. However, investigation indicated that AE signals were significantly attenuated within the cable joint. RFCTs were used to detect the discharge current flowing through the cable sheath. Where the detection sensitivity was low, a wavelet de-noising method was applied to process the RFCT signals and proved to be effective in increasing the measurement signal to noise ratio.
\end{abstract}

\section{INTRODUCTION}

$\mathrm{D}$ EFECTS within polymeric insulation of $\mathrm{HV}$ cables and cable joints such as voids, contaminants, or protrusions, can lead to partial discharge (PD) activity. It is important to monitor partial discharges within cables and joints and eliminate their cause, so that irreparable damage, possibly resulting in system failure, can be avoided. Cable joints are often installed on-site in less than ideal conditions. Defects that generate PD are more likely to exist within cable joints than in the cable itself.

PD activity generates both physical phenomena and chemical changes within the dielectric material. PD activity can cause the transmission of acoustic, electrical and optical energy. This can be detected and analysed using various methods to ascertain the presence of PD activity. Typical values obtained from these emissions are very small, typically signal magnitudes are often close to back-

Manuscript received on 17 July 2001, in final form 26 February 2002. ground noise levels and in the case of optical and chemical methods their detection suffers from a low sensitivity [1].

Conventional electrical PD testing has been used for many years to evaluate cable quality $[2,3]$. Such tests are generally performed off-line within a well-screened laboratory, under applied voltages higher than the normal operational voltage. The test circuit should be able to detect the permissible discharge quantity regulated for the test object. However, sometimes the purpose of PD measurement is to detect any deterioration of the apparatus during normal use, through on-line monitoring. Noise and interference can be severe under operating conditions and the measurement must be made at the normal operating voltage. Thus it is more difficult for on-line monitoring to detect small discharges during the initiation of insulation deterioration. However, when the deterioration reaches a certain level, warnings should be given in order to prevent the occurrence of breakdown. Apart from faults in cables and cable accessories due to long-term aging effects, 
spontaneous faults or breakdown can occur. It is important that on-line monitoring can detect new arising defects.

PD on-line monitoring for cable systems has been investigated by means of non-conventional electrical coupling approaches. These methods are based on detecting the high frequency characteristics of the discharge pulse. PDs can be considered as electromagnetic pulses travelling along the cable and the coaxial structure of the cable acts as a wave-guide for the electromagnetic wave. The semiconducting screens of the cable result in severe attenuation of the high frequency components within the discharge pulse.

There are various non-conventional coupling methods, for example:

(1) A coaxial cable sensor can be installed at the cable joint [4]; the sensor and the cable dielectric form a high frequency voltage divider consisting of the capacitance of the cable section and the sensor. It is possible to measure a discharge pulse by connecting a measuring impedance across an interruption in the metal earth screen of the cable $[5,6]$.

(2) Inductive coils can be clamped around the cable to detect the magnetic fields due to PD activity [7-9],

(3) Radio frequency current transducers (RFCT) can be used to detect the discharge current that flows through a conductor connecting the cable metallic earth screen to a grounding point. This technique has been widely applied to cable terminations $[10,11]$, as well as cable joints $[12,13]$.

(4) Directional coupling sensors can be placed on either side of a cable joint to detect partial discharges from within it [14-17].

For the capacitive coupler, directional coupling sensor and screen interruption methods it is necessary to remove a section of the outer metal earth screen to obtain a discharge measurement. The sheath interruption limits the short circuit current handling ability, especially for the screen interruption method where a measuring impedance is connected across the interruption. For on-line measurements, it is necessary to either place the measurement impedance across an inductor that can handle the short circuit current or to use over voltage protection in parallel with the impedance [5]. Inductive coupler detection of magnetic fields due to a discharge activity is more suitable for cables that have a helical shaped metal screen of individual wires. This is because the discharge pulses are forced to follow the helical wire resulting in a relatively high magnetic field outside the cable, which can be detected using a single-turn open loop inductive coupler without a magnetic core $[7,8]$. In solid shielded cables, however, the magnetic fields generated by PD activity outside the cable are much lower. In such cases a high frequency current probe with magnetic core can be applied around the cable [9]. Inductive sensors are easy to use and no modification of the cable is necessary. As the inductive sensor output signal magnitude is proportional to the rate of change of the magnetic flux through the sensor loop, a pulse which has had its higher frequencies attenuated is hard to detect and this limits the detection sensitivity. RFCTs detect the discharge current flowing to earth and are also easy to apply, requiring no modification of the cable system. The quantification or sensitivity check of a RFCT usually requires the injection of a calibration pulse into a cable termination. Consequently sensitivity checks are problematic for on-line measurements because the higher frequencies of the calibration pulse are significantly attenuated between the termination and the joint under test [13]. Directional coupling sensor techniques allow discrimination between PD internal to the cable joint and external pulses by analysing their direction of propagation. Careful optimisation is needed to combine good sensitivity with sufficient directivity [17]. A common problem with all of the non-conventional electrical PD detection methods is their susceptibility to electrical noise and interference.

When a PD occurs within the cable insulation, some of the discharge energy is emitted as acoustic energy. The percentage of the total discharge energy that is acoustic energy is very difficult to calculate, and it is material dependent. It is generally estimated to be very small and has been quoted as less than 1\% [18]. Acoustic measurements of PD activity rely on the detection of a pressure wave formed from a sudden deformation within the electrically stressed material. The acoustic emission (AE) approach has a distinct advantage of being immune to electrical interference; it is also easy to apply and consequently is a potential method for PD on-line monitoring. However, acoustic waves attenuate during propagation through cable materials and interfaces, and this may result in low detection sensitivity. Due to the attenuation of the acoustic wave it is difficult to quantify any PD on-line measurement in terms of apparent charge. However, acoustic emission techniques have been applied to PD detection and location in cables and cable accessories [19-22].

This paper investigates experimentally the application of several potential on-line methods, namely the use of capacitive couplers, acoustic emission sensors and radio frequency current transducers for PD detection within a $132 \mathrm{kV}$ prefabricated cable joint containing a known defect. The three methods were selected on grounds of cost, availability and the fact that they have been widely applied to PD detection within cable joints. Although each of the three methods has been applied individually to PD detection under different situations, a comprehensive investigation and comparison of these three methods has not yet been made. Furthermore, comparative information on the detection, location, quantification and identification of PD can he nhtained if several different methods 


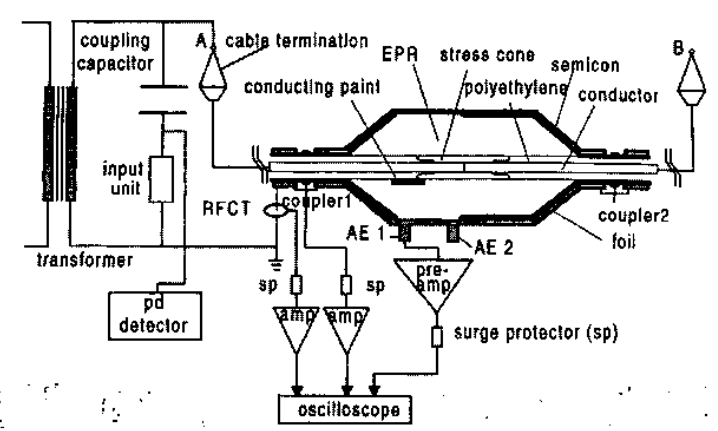

Figure 1. Schematic diagram of the PD test arrangement.

are applied simultaneously rather than individually to the joint under test:

\section{MEASUREMENT SYSTEM}

The measurement system is shown in Figure 1. Two 132 $\mathrm{kV}$ cable sections were connected by a prefabricated cable joint. The main insulation material of the cable joint is ethylene propylene rubber (EPR), whereas the cable insulation is cross-linked polyethylene (XLPE). The cable ends were connected to oil-filled cable terminations. Conventional electrical PD detection was applied to provide the discharge apparent quantity using a Robinson model 5 type 700 PD detector. The acoustic emission sensor was a piezoelectric device with a sensitivity of $-68 \mathrm{~dB}$ (ref. $10 \mathrm{~V} / \mathrm{Pa}$ ) and a resonant frequency of $150 \mathrm{kHz}$. The $\mathrm{AE}$ sensor output was comparatively small and therefore it was amplified using a preamplifier with frequency range of $20-1200 \mathrm{kHz}$ and $40 / 60 \mathrm{~dB}$ selectable amplification gain. A power supply unit, which can be operated by mains or by battery, provided the $28 \mathrm{~V} \mathrm{dc}$ supply voltage for the $\mathrm{AE}$ preamplifier. A wide band amplifier with frequency range of $0.01-500 \mathrm{MHz}$ and amplification gain of $24 \mathrm{~dB}$ was used to amplify the capacitive coupler signals. The surge protector $(\mathrm{sp})$ has a maximum operating frequency of $1 \mathrm{GHz}$ and a response time less than $10 \mathrm{~ns}$. The surge protector was used to protect the measuring system from any possible over-voltage during the PD tests. The digital oscilloscope has a maximum sampling rate of $5 \mathrm{G}$ Samples/s and analogue bandwidth of $1 \mathrm{GHz}$. In addition to the acoustic emission sensor and the capacitive coupler, two ferritecore radio frequency current transducers with frequency response of $2.5 \mathrm{kHz}-150 \mathrm{MHz}$ or $10 \mathrm{kHz}-200 \mathrm{MHz}$ were also applied. A Pentium II $233 \mathrm{MHz}$ computer was used to post-process the measurement data. The signal processing and analysis software were developed using a commercial mathematics programming environment.

Figure 2 shows the arrangement of capacitive coupler, acoustic emission sensor and RFCT. The acoustic emission sensor was bound onto the cable joint by mechanical pressure and silicon grease was applied between the acoustic emission sensor and the joint surface, to reduce the air gap that causes acoustic interface attenuation. In order to detect the discharge current flowing to earth, the

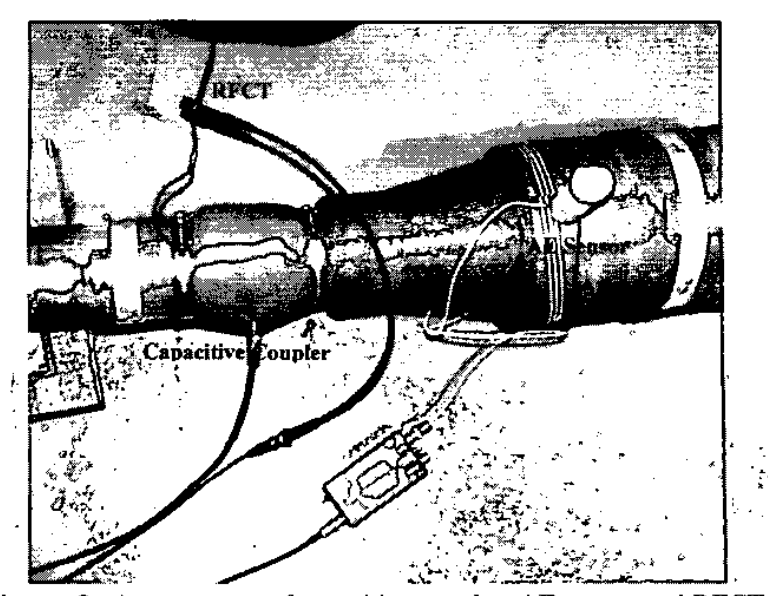

Figure 2. Arrangement of capacitive coupler, AE sensor and RFCT.

RFCT was placed around a conductor that connected the cable sheath to the system earth. Capacitive couplers were installed on the cable section close to the cable joint. To reduce the influence of electromagnetic interference, the capacitive couplers were screened.

Prior to creating a fault within the joint, the measurement system was thoroughly tested to ensure that it was discharge free up to $160 \mathrm{kV}$. The joint was then dismantled and conducting paint in the shape of a ' $v$ ' was installed on top of the cable XLPE between the cable joint stress cone (conductor) and the cable joint outer semiconducting layer. This acted as the PD source within the cable joint.

\section{CAPACITIVE COUPLER MEASUREMENTS \\ 3.1 CAPACITIVE COUPLER AND EQUIVALENT CIRCUIT}

The capacitive coupler is shown in Figure 3. A $100 \mathrm{~mm}$ long section of the cable metal sheath was removed, and $40 \mathrm{~mm}$ wide tin tape was wrapped around the exposed cable outer semicon screen. The tin tape acts as the coupler sensor. The use of different sensor materials such as aluminium were also investigated, results obtained indicate that the coupler output magnitudes and waveforms are similar. The coupler does not disturb the insulation system because the relatively high potential electrode of

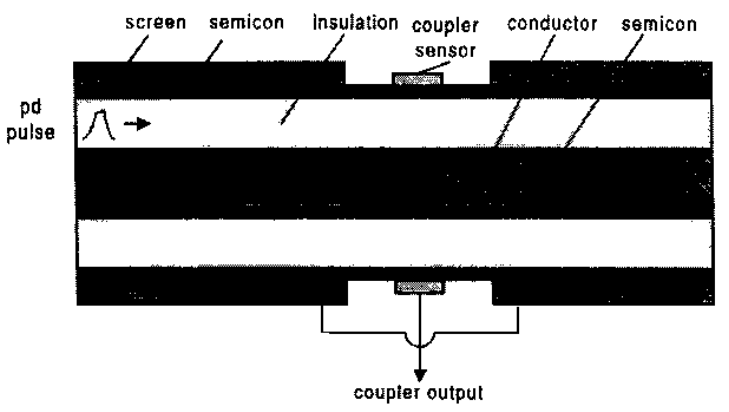

Figure 3. Diagram of a capacitive coupler. 


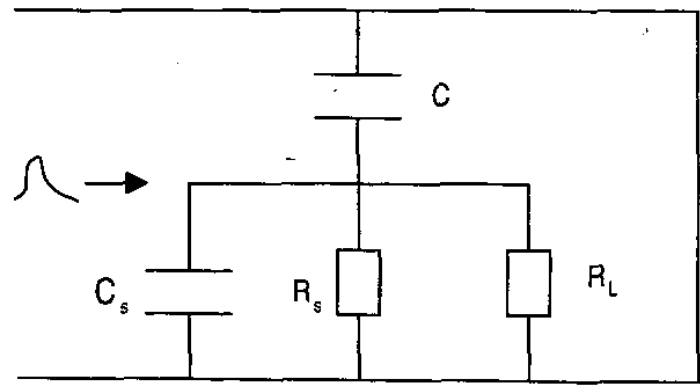

Figure 4. Equivalent circuit of thc capacitive coupler.

the coupler is attached on top of the outer semicon layer which serves as the power frequency ground, leaving the internal field distribution of the cable unaffected. The lower potential electrode of the coupler is defined by the outer metallic ground screen because of the lower conductivity of the semicon layer which has complex frequency dependent characteristics.

The equivalent circuit model of the coupler is shown in Figure 4. $C$ is the coupler capacitance, which is dependent on the length of the coupler and the cable capacitance per metre $C_{0} . R_{s}$ is the impedance between the coupler sensor and the metal sheath, which depends on the resistance of the semicon layer; $C_{s}$ is the stray capacitance between the coupler sensor and the sheath; and $R_{L}$. is the detecting impedance which is the input impedance of the measuring circuit. $C_{0}$ can be determined using

$$
C_{0}=\frac{2 \pi \epsilon_{0} \epsilon_{r}}{\ln \frac{D_{1}}{D_{0}}}
$$

Where $D_{1}$ is the diameter of the insulation (excluding the thickness of the cable outer semicon screen); $D_{0}$ is the diameter of the conductor (including the thickness of the inner semicon screen); $\epsilon_{0}$ is the dielectric constant in vacuum, which is $8.85 \mathrm{pF} / \mathrm{m}$; and $\epsilon_{r}$ is the relative dielectric constant of the insulation material. It should be noted that the approximate coüpler equivalent circuit has not taken the influence of the inner and outer semicon layers into consideration. It is well known that the cause of signal attenuation and distortion in coaxial power cables is mainly due to the losses caused by the frequency dependant semiconducting screens. The dielectric constant of the semicon layers of XLPE cable generally decreases with increased frequency [23]. However, it should also be noted that the impedance of the dielectric is much greater than that of the semiconducting layers. The dielectric is much thicker and therefore has a much lower capacitance per unit length than the semicons: The dielectric has a much lower dielectric constant than the semicons (generally from 10 's to 100 's), and the dielectric has much lower conductivity than the semiconducting layers [24]. Consequently the discharge current causes a voltage drop mainly across the cable dielectric. Due to the short length of the coupler cable section (100 $\mathrm{mm}$ in this case), the influence of the semicon layers on the sensor signal characteristic is also small. Furthermore, the dielectric constant of the insulating material, such as polyethylene, varies by less than $0.5 \%$ over the frequency range from $60 \mathrm{~Hz}$ to $10 \mathrm{GHz}$, therefore the cable distributed capacitance is generally a true - "constant"[25].

For long cable sections and cable joints rather than the short coupler section, however, the high frequency attenuation caused by semicon layers does play an important role. For example, it affects the detection sensitivity of the couplers, as discharge pulses will decrease in magnitude and distort in waveform with longer rise time and width, when it reaches the coupler.

\subsection{QUANTIFICATION OF THE CAPACITIVE COUPLER}

Quantification or sensitivity checks can be achieved by injecting calibration pulses into the system and comparing the measured amplitude from the detector output with the injected charge. This may lead to quantification results, which not only depend on the charge of the injected pulse, but also on its wave shape, and consequently the frequency spectrum of the pulse which reaches the detector. It has also been found that the sensor characteristic has a major effect on the sensor response [17]. Several çalibration methods for non-conventional PD measuremént systems have been proposed [17].

\subsubsection{QUANTIFICATION FROM A CABLE TERMINATION}

Step waves from a pulse generator, in series with a capacitance of $6 \mathrm{pF}$, were injected into the cable termination between the HV conductor and the ground. The step wave had a rise time of 1 ns but different amplitudes were applied. The injected charge can be approximately taken as the step wave amplitude multiplied by the $6 \mathrm{pF}$ capacitance. Figure 5 shows the relationship between the coupler 1 output amplitude and the injected charge. A nearly

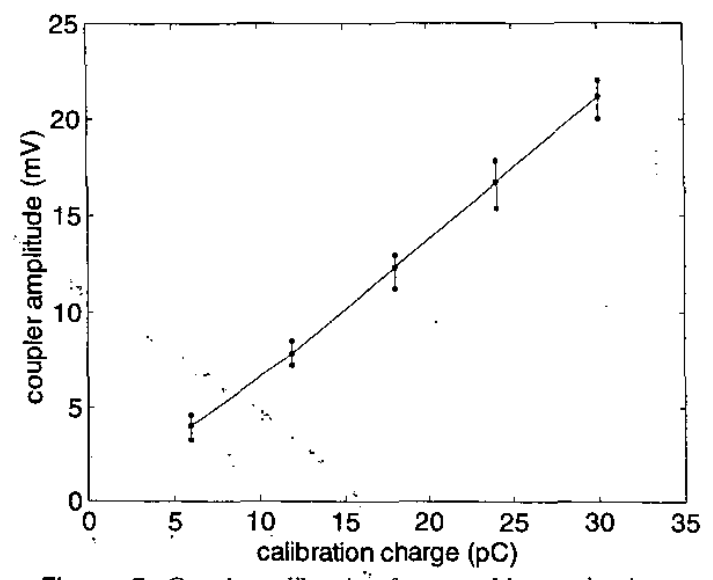

Figure 5. Coupler calibration from a cable termination. 
linear relationship exists, and the quantification result is approximately $0.7 \mathrm{mV}(\mathrm{pC})^{-1}$. The nearly linear relationship might be explained as the coupler transfer impedance is constant and the injected charges have the same frequency characteristics but different magnitudes.

Attenuation within the cable system may reduce the calibration signal and alter its wave shape before it reaches the coupler, which will consequently introduce quantification errors. The nature of this method makes it unsuitable for on-line calibration of in-service cable joints.

If an extra cable joint, similar to the one to be assessed, is available, it would be possible to use it for quantification by cutting its cable very close to the joint and injecting a voltage pulse in series with a known capacitance directly into the cable core [17]. A coupler installed on the other side of the extra cable joint can then measure the calibration pulse. In this way the attenuation or distortion of the injected pulse would be less significant compared with quantification through a distant cable termination. Obviously this method still is not an on-line quantification approach for the capacitive coupler.

\subsubsection{QUANTIFICATION ACROSS TWO CAPACITIVE COUPLERS}

If two capacitive couplers are installed on either side of the cable joint, one can be used to inject a calibration pulse that can be detected using a second coupler. From the equivalent circuit for capacitive coupler, the principle of this quantification method is detailed in Figure 6. The coupler capacitance $C$ can be calculated using Equation 1. For this experiment, the capacitance of the $132 \mathrm{kV}$ cable section was calculated to be $270.6 \mathrm{pFm}^{-1}$.

Considering Figure 6 , if the influences of the stray capacitance and resistance are ignored, the injected charge can be estimated as the pulse generator output magnitude is multiplied by capacitance $C$. A step wave from the pulse generator with a magnitude of $5 \mathrm{~V}$ and rise time of $1 \mathrm{~ns}$, was injected into coupler 2 , for a range of coupler 2 sensor lengths equivalent to different cable capacitances. Figure 7 shows that a generally proportional relationship exists between the output magnitude of coupler 1 and the sensor length of coupler 2 .

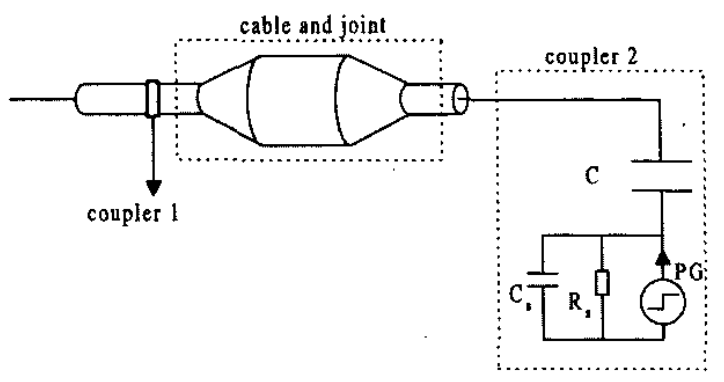

Figure 6. Principle of calibration through another coupler.

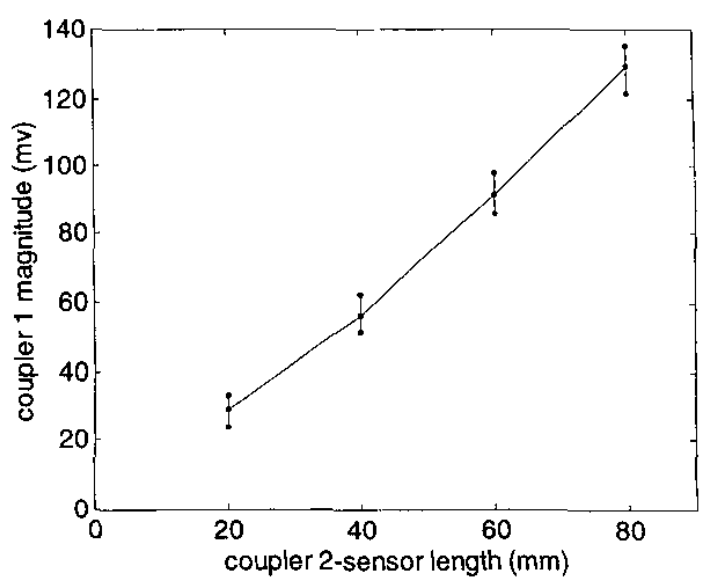

Figure 7. Influence of coupler sensor dimensions on calibration.

Step waves from the pulse generator, with a 1ns rise time but with different amplitudes, were then injected into coupler 2. In this case, the length of the coupler sensor was $40 \mathrm{~mm}$ equivalent to a cable capacitance of $10.8 \mathrm{pF}$. Figure 8 shows the relationship between the output amplitudes of coupler 1 and the approximate injected charge: A nearly linear relationship exists, and the quantification result is about $0.97 \mathrm{mV}(\mathrm{pC})^{-1}$.

Figure 9 shows the coupler 1 output waveform due to a step wave with rise time of $1 \mathrm{~ns}$ and magnitude of $5 \mathrm{~V}$ injected into coupler 2 . There are reflected pulses in the coupler output signal, and investigation indicates that these reflections occur at the two cable terminations.

In principle this method can only provide an approximate quantification result, as the calibration signal is injected into a coupler rather than the cable core. However, it might provide the only acceptable practical solution to on-line capacitive coupler quantification.

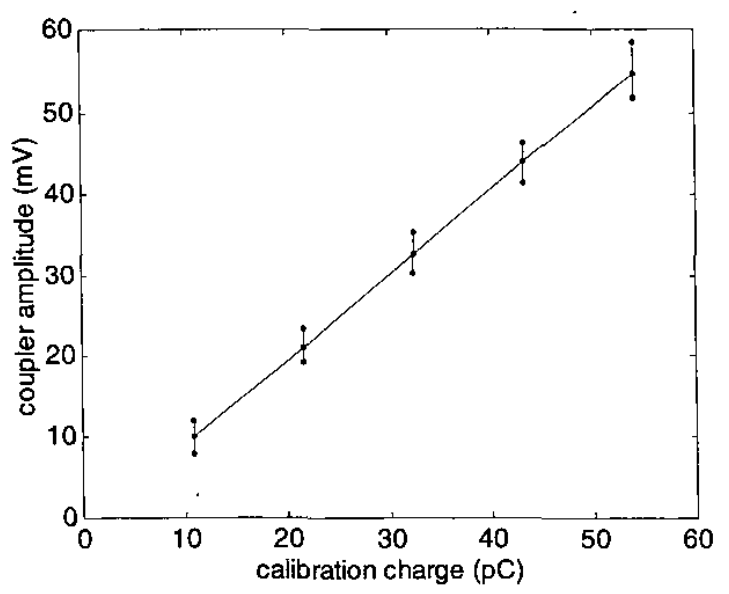

Figure 8. Calibration results using another coupler. 


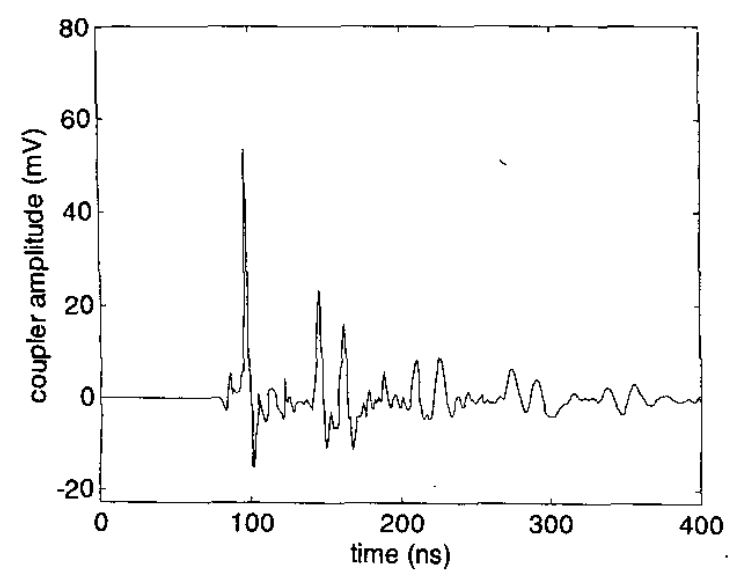

Figure 9. Coupler response to a calibration pulse from another coupler.

\subsection{INTERNAL DISCHARGE DETECTION USING CAPACITIVE COUPLER}

A HV was applied to the joint in order to generate PDs due to the presence of conducting paint within the joint. The PD inception voltage was found to be $34 \mathrm{kV}$. Figure 10 shows the coupler 1 output signal and the relevant discharge quantity obtained by conventional electrical detéction. Discharges with magnitude greater than $3 \mathrm{pC}$ can be detected by the capacitive coupler. In this case the sensitivity of the capacitive coupler output was found to be around $1.6 \mathrm{mV}(\mathrm{pC})^{-1}$. Considering the quantification result of $0.7 \mathrm{mV}(\mathrm{pC})^{-1}$ from the cable termination and 1.0 $\mathrm{mV}(\mathrm{pC})^{-1}$ from injection via a second coupler, errors exist for either of the proposed methods. This may be due to the difference between the real PD pulses and the injected pulses with known pulse characteristics. The attenuation is different due to the different pulse propagation paths for three cases. In theory, the pulses that are in-
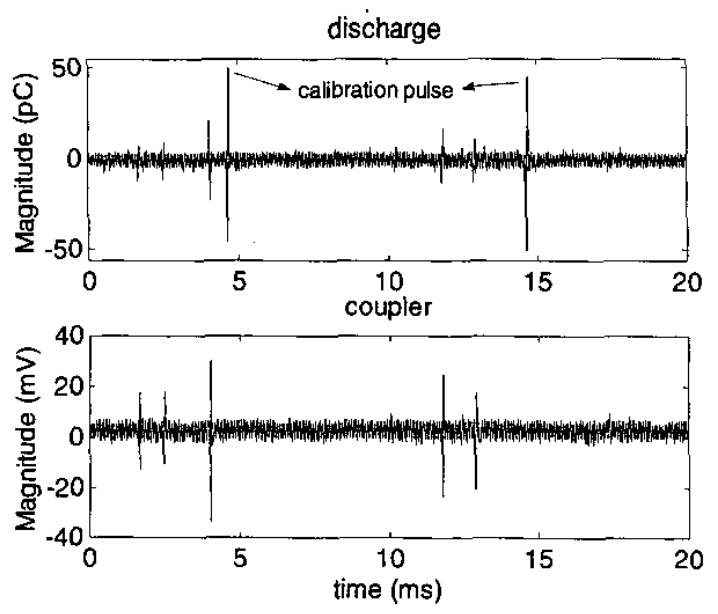

Figure 10. Practical coupler outputs due to partial discharges.
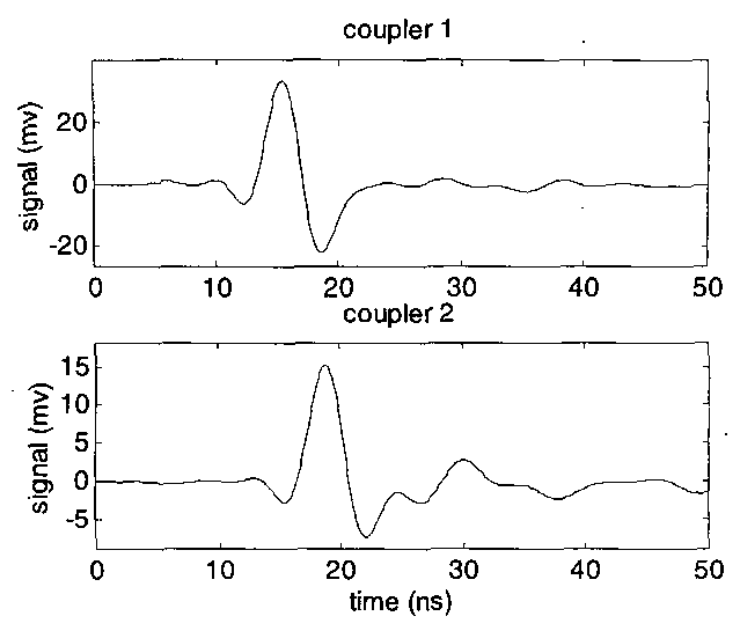

Figure 11. Time of flight for couplers installed at the cable joint system.

jected into the cable termination and travel through the termination and cable section suffer the highest attenuation; the pulses injected into another coupler travelling through the entire cable joint are less attenuated; and the internal discharge pulses travelling through part of the cable joint are the least attenuated. This agrees with the obtained sensitivity measurements and test results.

In order to determine the discharge pulse travelling speed within the cable section/joint a step wave was injected into cable termination A (Figure 1), and the time delay between coupler 1 and 2 was found to be $6.7 \mathrm{~ns}$. Given that the distance between the couplers is $1.05 \mathrm{~m}$, the discharge pulse travelling speed for this cable/joint arrangement was estimated to be $0.157 \mathrm{~m}(\mathrm{~ns})^{-1}$. Figure 11 shows the output of two couplers seeing the same discharge pulse. Coupler 1 leads coupler 2 with a time flight of $3.4 \mathrm{~ns}$. This information can be used to provide an accurate estimate of the location of the PD activity.

Figure 12 shows the coupler 1 output waveform and frequency spectrum. The frequency components are within the range of 10 to $300 \mathrm{MHz}$. There are some reflections within the coupler waveform. The discharge pulse from the PD site travelled along the cable in opposite directions. The coupler detected the pulse travelling to the left (pulse 1 in Figure 12). The same pulse continued to travel to the left and was reflected by cable termination $A$. The reflected pulse then travelled to the right and was detected again by the coupler (pulse 2 in Figure 12). The original discharge pulse travelling to the right from the PD site was reflected by cable termination $B$, the reflected pulse travelled back to the left and later was detected by the coupler (pulse 3 in Figure 12). Analysis of the time of flights indicate that they agree with practical individual travelling distances, considering the known discharge pulse travelling speed along the cable system. The 

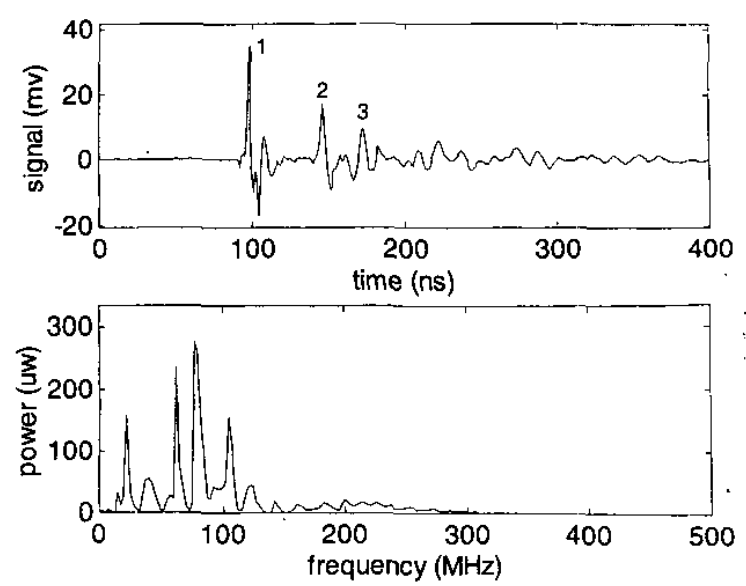

Figure 12. Coupler waveform and spectrum for a PD within cable joint.

reflected pulses would continue to travel along the cable and will be further reflected by the cable terminations. However, due to the propagation attenuation and reflection, further reflections are not clearly visible.

\subsection{CORONA DISCHARGE DETECTION USING CAPACITIVE COUPLER}

Corona discharges were generated in order to study the capacitive coupler response to them. A pair of metal scissors were hung from cable termination A upper electrode (high potential) and pointed at the bottom electrode (ground). In this case, the corona inception voltage was 21 $\mathrm{kV}$ (below the level required to generate $\mathrm{PD}$ within the joint) and the discharge level was $250 \mathrm{pC}$. A similar corona discharge measurement was also carried out, using aluminium wire with diameter of $1 \mathrm{~mm}$. In this case the corona inception voltage was found to be $14 \mathrm{kV}$ and the discharge level was only $30 \mathrm{pC}$. Figure 13 shows the measured signal relating to the first case from coupler 1 over one voltage cycle, and is typical of corona discharge. Figure 14 shows a single corona discharge waveform and its relevant frequency spectrum. Compared with the results

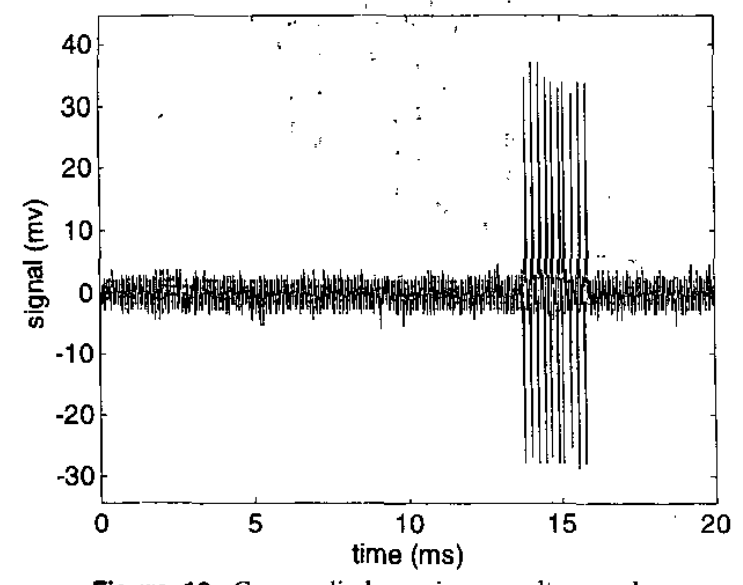

Figure 13. Corona discharge in one voltage cycle.
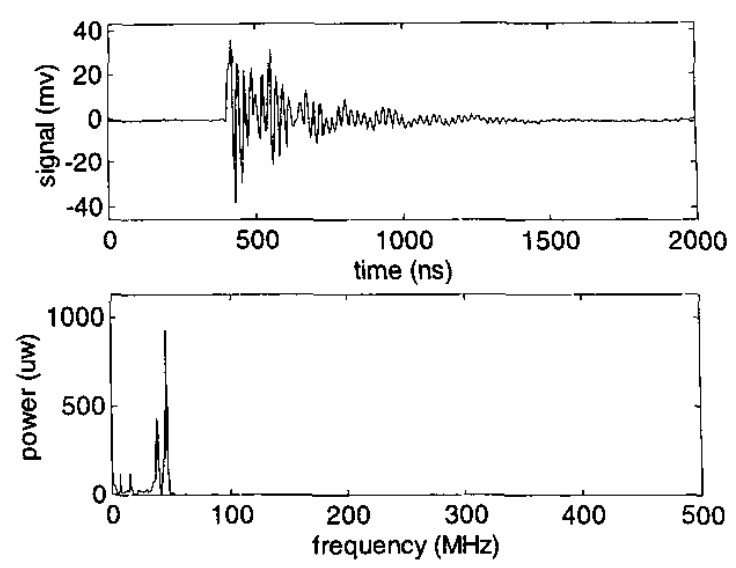

Figure 14. Capacitive coupler response to corona discharge.

shown in Figure 12, the coupler output due to corona discharge has a longer time duration and contains lower frequency components. Figure 15 shows the time based signals for two coupler outputs due to corona discharge. Comparing the two traces reveals that the source of corona discharge is from the left side of coupler 1 rather than between the two couplers.

The obtained results indicate that if two capacitive couplers are placed on either side of the cable joint under test, then the discharge pulse shape, its relevant frequency spectrum, and time of flight can be used to distinguish between PD pulses from within the cable joint and external noise.

\section{ACOUSTIC EMISSION MEASUREMENTS}

The source of corona discharge was removed and acoustic emission sensors were applied to detect PD activity within the cable joint. Experimental investigation indi-
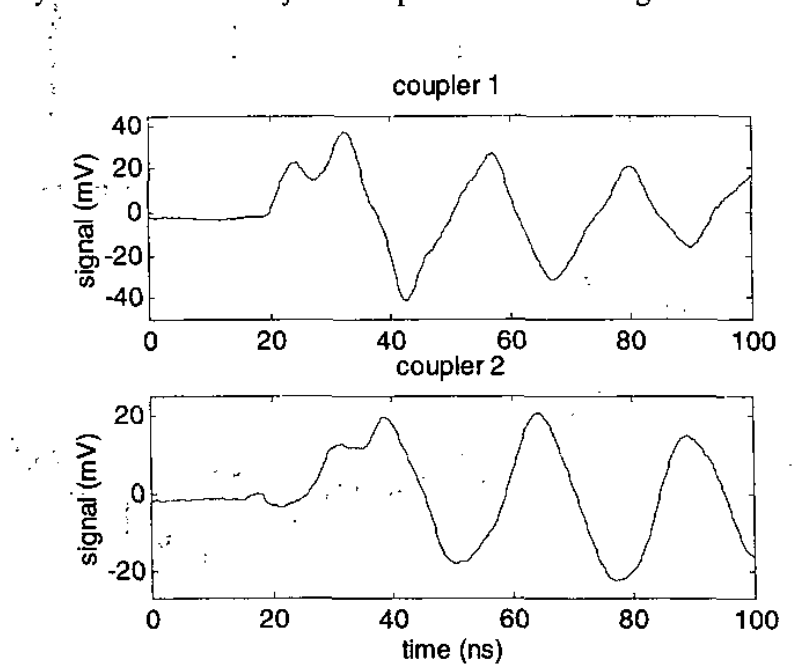

Figure 15. Time of flight for two coupler outputs due to corona discharge. 

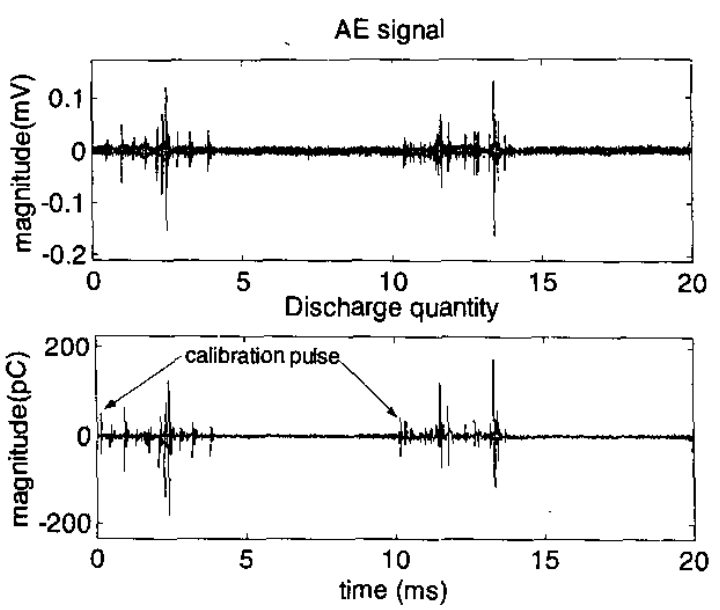

Figure 16. Discharge acoustic \& electric signals.

cates that the $\mathrm{AE}$ sensor is immune to external electrical interference. Figure 16 shows the discharge acoustic and electric signals at an applied voltage of $50 \mathrm{kV}$. The electrical signals were obtained by conventional electrical detection. Partial discharge produces both acoustic and electrical signals, and they relate to each other with a time of flight which is the time required for the acoustic emission signal to travel from the PD site to the AE sensor.

To investigate the AE signal attenuation with the cable joint, a second $\mathrm{AE}$ sensor was placed $85 \mathrm{~mm}$ away from $\mathrm{AE}$ sensor 1, as shown in Figure 1. When the discharge level was $15 \mathrm{pC}, \mathrm{AE}$ sensor 1 can detect the acoustic emission signals but AE sensor 2 cannot (Figure 17a). When the test voltage was increased and a discharge level of 150 pC was obtained, both sensors could detect the acoustic signals, but with different sensitivities (Figure 17b). The results indicate that the $\mathrm{AE}$ signal attenuation is significant within the cable joint, which reduces the PD acoustic emission detection sensitivity. In addition the attenuation is highly dependent on the materials used in the cable joint construction. However, in situations where electric noise is severe, acoustic emission methods may be more effective to detect the presence of PD activity rather than electrical couplers.

The distance from the PD site to the acoustic emission sensor can be calculated as the multiplication of the acoustic emission propagation time and velocity. Figure 18 shows the discharge electric pulse and two corresponding acoustic emission sensor output signals. In this case, the discharge electric pulse is obtained by conventional electrical detection. The time of flight is $45 \mu$ s between $\mathrm{AE}$ sensor 1 and the discharge pulse and $93 \mu$ s between $\mathrm{AE}$ sensor 2 and the discharge pulse. Given that the acoustic emission wave propagation velocity is $1546 \mathrm{~ms}^{-1}$ in ethylene propylene rubber [18], the obtained results agree with the practical geometric distances of $68 \mathrm{~mm}$ and
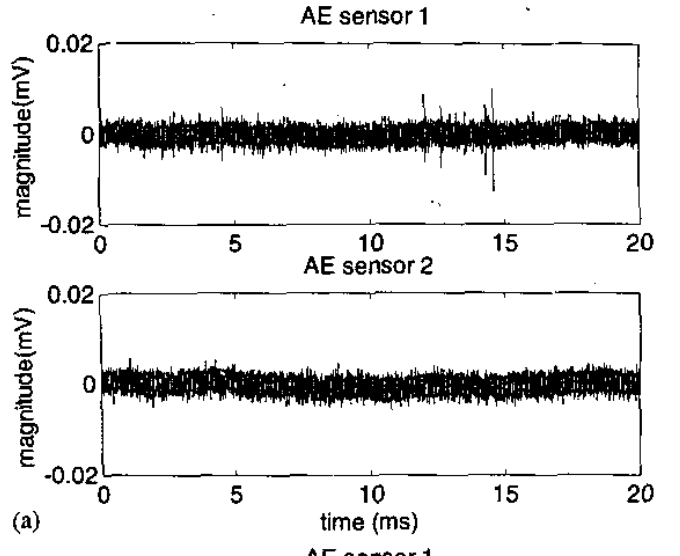

(a)
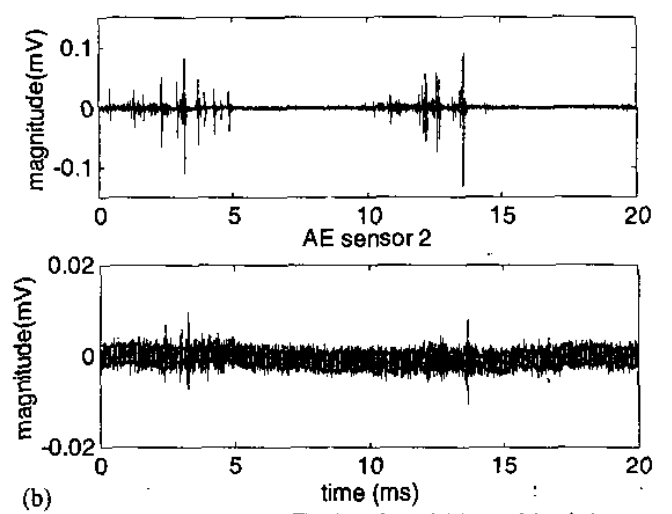

Figure 17. Attenuation of $\mathrm{AE}$ signals within cable joint. a, discharge level $15 \mathrm{pC} ; \mathrm{b}$, discharge level $150 \mathrm{pC}$.

$146 \mathrm{~mm}$ respectively of the two AE sensors from the PD site.

AE sensors have been applied to PD location in power transformers. Transformers have a relatively regular geometry compared to a cable and may be approximated as a rectangular object. Consequently PD location in threedimensional space can be investigated. If three or more $\mathrm{AE}$ sensors are installed at different positions, then the
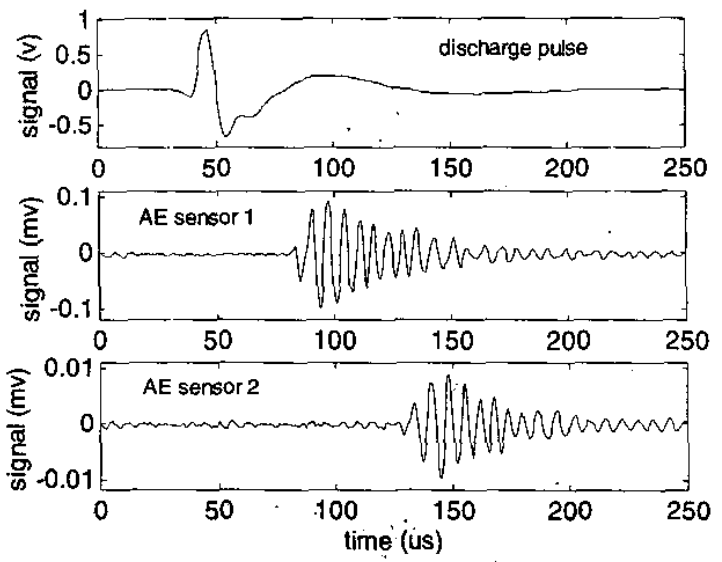

Figure 18. Time of flight measurements using $\mathrm{AE}$ sensors. 
three-dimensional distances from the PD site to different sensors with known co-ordinates can be related using the acoustic wave velocity and time of flight. Thus a series of equations can be obtained, which can be solved using a least squares algorithm and an iteration method [26]. Theoretically a similar location method can be applied to a cable joint. However, compared to a power transformer, the cable joint has much smaller dimensions and dissimilar acoustic properties. Different insulation materials such as EPR and XLPE, semiconducting screen layers and conductors influence the acoustic wave propagation path and velocity. Therefore, in practice a convenient and feasible location method might be achieved by applying two $\mathrm{AE}$ sensors and moving them along the cable joint surface until the peak magnitude signal is obtained and the corresponding time of flight is a minimum. If both signals are identical then the plane of the PD site is equidistant from the two sensors.

A combination of PD electrical and acoustic emission detection can also be used for discriminating between partial discharges and noise. For partial discharges, the electric pulse leads the acoustic pulse with a time of flight, which corresponds to the propagation time of the acoustic wave from the PD site to the sensor (Figure 18). For noise, one of the three cases may apply: the electric pulse has no related acoustic pulse; the acoustic pulse has no related electric pulse; or no time of flight exists between the electric and acoustic pulses. Apart from conventional electrical detection, discharge electric pulses can also be obtained using a non-conventional electrical sensor such as an inductive current transducer.

\section{RFCT MEASUREMENT AND WAVELET DE-NOISING}

In order to detect any discharge current flowing to ground a ferrite-core radio frequency current transducer with a small aperture $(5 \mathrm{~mm})$ was clamped around the conductor connecting the cable sheath to the system earth. The RFCT has a frequency range of $2.5 \mathrm{kHz}$ to $150 \mathrm{MHz}$. Figure 19 shows the measured signals. The RFCT output was amplified by a wide band amplifier which has a frequency range up to $500 \mathrm{MHz}$ and gain of $24 \mathrm{~dB}$. The discharge quantity was obtained by the conventional electrical detection. In this case the signal to noise ratio (SNR) was found to be low, resulting in a low detection sensitivity. Only discharges above $90 \mathrm{pC}$ were detectable. The SNR can be improved using appropriate filtering techniques.

Many existing discharge signal de-noising methods are based on the fast Fourier transform (FFT), where analysis of PDs is obtained using frequency information. The spectra of noise can be seen as sharp lines with peak values at respective frequencies, which can be minimised by applying a suitable threshold. An inverse transform is then applied to obtain filtered time-based data. However, a dis-
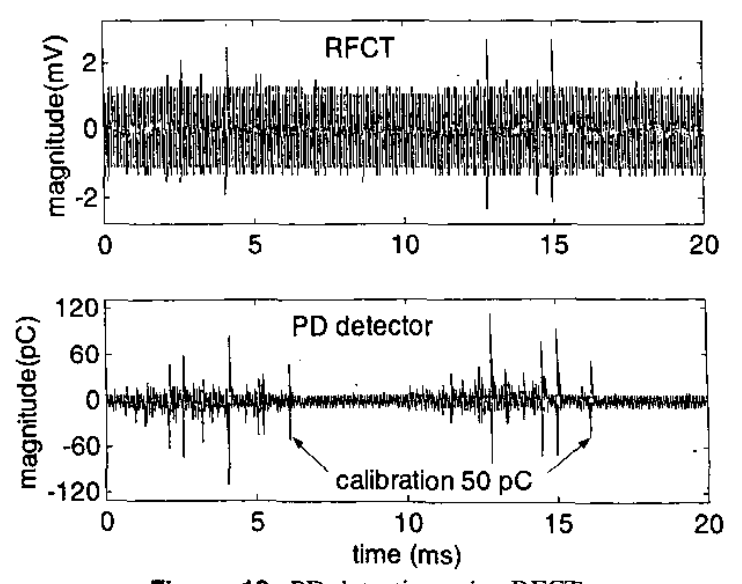

Figure 19. PD detection using RFCT.

charge pulse covers a wide range of frequencies. Therefore, when noise at certain frequencies is reduced, some components of PD signal itself may be lost through conventional filtering. Moreover, the FFT algorithm is based on a deterministic data set. De-noising methods developed using the FFT are therefore limited in processing PD signals that are stochastic in nature [27].

Due to the limitation of the conventional de-noising methods using Fourier transforms, the wavelet transform is becoming popular in signal processing. Discharge signals and random noise may have different behaviour under a wavelet transform. For filtering, the signal is decomposed into a series of approximation and detail coefficients at various scales. Thresholding of wavelet coefficients is then conducted by retaining the wavelet coefficients corresponding to discharge events and discarding all noise coefficients. Finally PD signals are reconstructed using the modified approximation and detail coefficients. For wavelet de-noising, the selection of the wavelet basis function (wavelet family) and the selection of thresholding method are important. There are various wavelet families, such as Haar, Daubechies, Biorthogonal, Cöiflets, Symlets, Morlet, Mexican Hat, and Meyer wavelet. Usually one of two thresholding methods are used: "soft" or "hard" [27].

Among all wavelet families Daubechies wavelet combined with soft thresholding have been widely applied to PD-related studies $[28,29]$. Work by the authors has also been undertaken on the performance of different wavelet families and thresholding methods. Daubechies $\mathrm{db} 2$ wavelet ( 2 denotes the wavelet order)' and the soft thresholding method have been found to provide the best results. Figure 20 shows the RFCT signals after de-noising and the relevant discharge quantity obtained by conventional PD detection. The SNR has been improved, and discharges of $30 \mathrm{pC}$ can now be recognised. Certainly wavelet post-processing is not only applicable to RFCT measurements. It could also be applied to other PD de- 

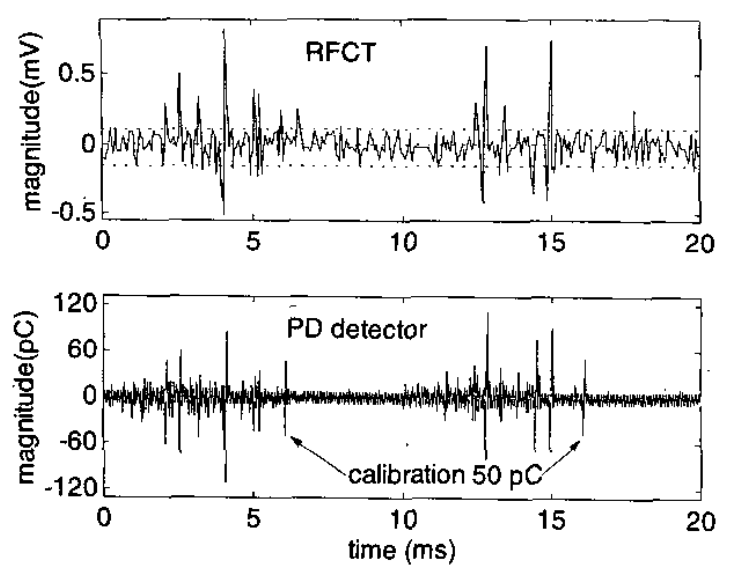

Figure 20. Wavelet de-noising of RFCT signals.

tection approaches where levels of background noise are a significant problem.

An EMCO $^{\circledR}$ ferrite core RFCT (model 93686-5) was also applied. It has a frequency range of $10 \mathrm{kHz}$ to 200 $\mathrm{MHz}$, and an aperture of $67 \mathrm{~mm}$. The RFCT is a split type, therefore it can be applied without interrupting the circuit. Figure 21 shows the detected PD signals. The RFCT output was also amplified using the same wide band amplifier as the small aperture RFCT. The discharge quantity was obtained by conventional electrical detection. The detection sensitivity for this RFCT is about 20 $\mathrm{pC}$, which is much higher than the result obtained using the small aperture ferrite RFCT. Consequently in this case the developed wavelet de-noising technique was not considered to be necessary.

Figure 22 shows a RFCT. detected discharge waveform and its relevant power spectrum. The frequency components extended to $100 \mathrm{MHz}$ but were dominant below 40 $\mathrm{MHz}$.

The RFCT was also used to detect corona discharge. A sharp point was placed at the high voltage coupling capac-
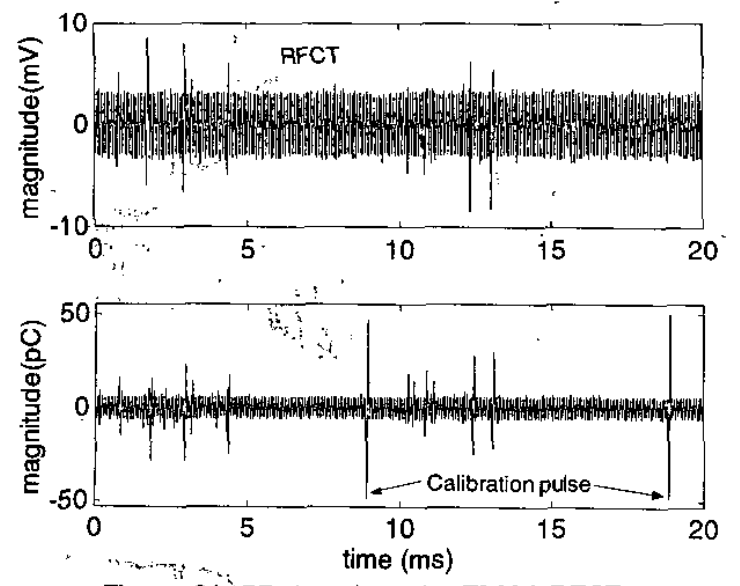

Figure 21. PD detection using EMCO RFCT.
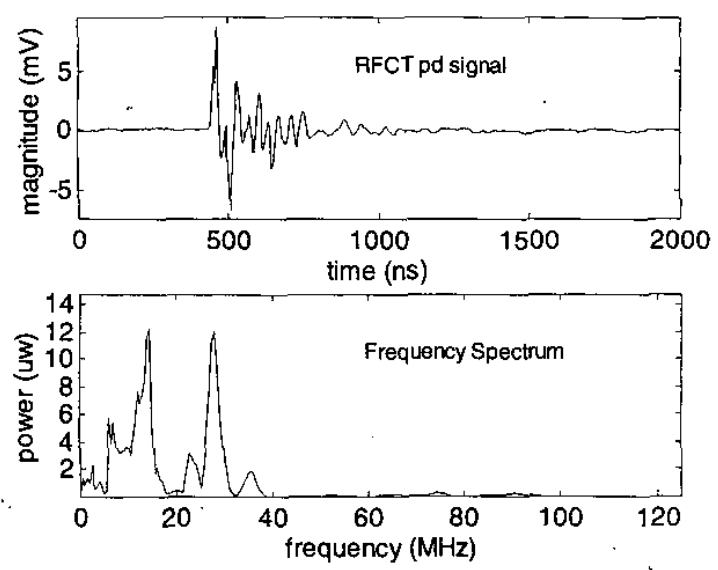

Figure 22. RFCT PD signal and frequency spectrum.

itor and pointed toward the earth. In this case, the corona inception voltage was $15 \mathrm{kV}$ and the discharge level was $35 \mathrm{pC}$. Figure 23 shows the RFCT detected signals and the discharge quantity obtained by the conventional electrical detection, which indicates the typical characteristics of corona discharge.

\section{CONCLUSIONS}

A comprehensive investigation and comparison of applying capacitive couplers, acoustic emission sensors and radio frequency current transducers to PD detection within XLPE cable joints has been undertaken. Quantification or sensitivity checks for a capacitive coupler was realised by injecting a very high frequency calibration pulse into either the cable termination or into another capacitive coupler. Quantification using two capacitive couplers may provide the only practical solution to on-line quantification. An equivalent circuit for this quantification method has been established.
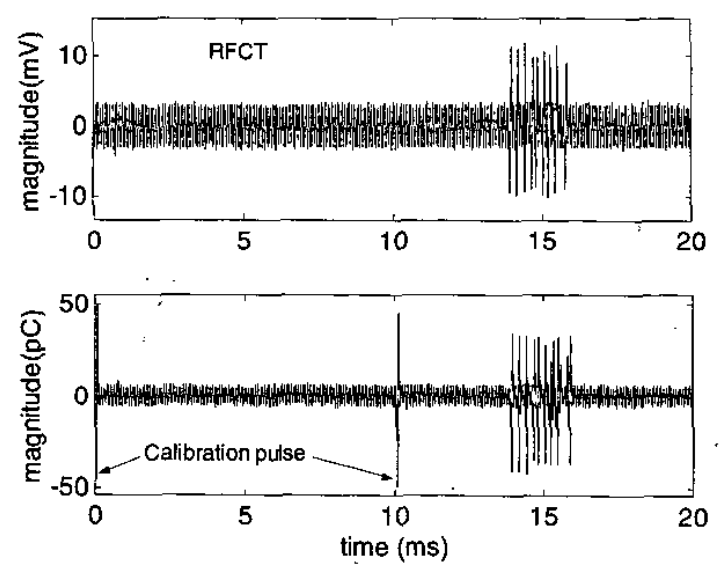

Figure 23. Corona discharge in one voltage cycle detected by RFCT. 
Discharge levels of $3 \mathrm{pC}$ can be detected using a capacitive coupler, indicating high detection sensitivity using this method. Location of the PD site can be realised by analysing the time of flight from two or more capacitive couplers. Obtained results indicate that the coupler output pulse shape, relevant frequency spectrum, and time of flight traces may be used to distinguish between internal discharges from the cable joint and external sources of noise. The capacitive coupler can be influenced by external interference; thus it needs to be well shielded and if necessary obtained signals might require filtering. A disadvantage of this approach is that part of the cable metal screen has to be removed in order to implement the coupler sensor.

PDs produce both electric pulses and acoustic emission waves, and they relate to each other with a small time delay that is the required time for the $\mathrm{AE}$ wave to travel from the PD site to the sensor. The acoustic emission approach has the advantage of being free from electrical interference, especially when PD measurements are carried out on site. Acoustic location of a PD site was investigated by analysing the time of flight between the discharge electric pulse, and different AE sensor outputs. The major disadvantage of this approach is the significant attenuation of the AE signal within the cable joint, which influences the $\mathrm{AE}$ detection sensitivity. The attenuation also makes it difficult to quantify $\mathrm{AE}$ signal magnitude $(\mathrm{mV})$ with discharge apparent quantity (pC). Initially a ferrite core RFCT with a small aperture was used to detect the discharge current flowing through it. Obtained results indicate that the detection sensitivity is low, as only discharges above $90 \mathrm{pC}$ were detectable. A wavelet transform was then applied to process the obtained signals. Through wavelet de-noising the SNR was improved such that discharges of $30 \mathrm{pC}$ could be observed. An EMCO ${ }^{\circledR}$ split ferrite RFCT with a large aperture was also applied to detect the discharge current. Results indicated that in this case the detection sensitivity increased to $20 \mathrm{pC}$ without the need for additional signal processing. The RFCT is also influenced by external interference. However, this method has an obvious advantage that it is very easy to apply and no modification of the cable/joint system is required.

The results of this work should assist in the development of a comprehensive and effective PD on-line monitoring system for cables and cable accessories.

\section{ACKNOWLEDGMENT}

The authors would like to acknowledge the financial support from National Grid, and their permission for the publication of this work.

\section{REFERENCES}

[1] I. J. Kemp, "Developments in Partial Discharge Plant-Monitoring Technology", International Conference on Partial Discharge, pp. 52-55, University of Kent, Canterbury, UK, 1993.
[2] F. H. Kreuger, "Discharge Detection in High Voltage Equipment", Temple Press Books Ltd, London, 1964.

[3] IEC Publication 270, "Partial Discharge Measurements", 1981.

[4] C. Henningsen, K. Polster, B. Fruth and D. Gross, "Experience with an On-line Monitoring System for $400 \mathrm{kV}$ XLPE Cables", Record of IEEE T\&D Conf., pp. 515-520, Los Angeles, USA, 1996.

[5] E. Pultrum and M. Van Riet, "HF Partial Discharge Detection of HV Extruded Cable Accessories", JICABLE, pp. 662-665, 1995.

[6] E. Ildstad, H. Faremo, O. Lillevik and D. Linhjell, "Detection and Location of Partial Discharges Sources in XLPE Cables", Proc. 10th ISH, Montreal, Canada, 1997.

[7] K. Fukunaga, M. Tan and H. Takehana, "New Partial Discharge Detection Method for Live UHV/EHV Cable", IEEE Trans. EI, Vol. 27, pp. 669-674, 1992.

[8] H. Bidhendi and Q. Su, "Partial Discharge Location for Cables with Helical Earth Screens", Proc. 10th ISH, Montreal, Canada, 1997.

[9] N. H. Ahmed and N. N. Srinivas, "On-line Partial Discharge Detection in Cables", IEEE Trans. DEI, Vol. 5, pp. 181-188, 1998.

[10] N. Ahmed, O. Morel and N. Srinivas, "Partial Discharge Measurement in Transmission-Class Cable Terminations", Proc. IEEE Transmission and Distribution Conf., pp. 2-7, 1999.

[11] H. N. Bidhendi and Q. Su, "A New Partial Discharge Location Technique for Power Cables Based on the Extension of the Characteristics Impedance", Proc. 10th ISH, Montreal, 1997.

[12] R. J. Jackson, A. Wilson and D. B. Gicsner, "Partial Discharges in Power Cable Joints: Their Propagation Along a Crossbonded Circuit and Methods for their Detection", IEE Proc. Vol. 127, Pt. C, pp. 420-429, 1980.

[13] Heizmann, T. Aschwanden, H. Hahn, M. Laurent and L. Ritter, "On-Site Partial Discharge Measurements on Premoulded Cross-Bonding Joints of $170 \mathrm{kV}$ XLPE and EPR Cables", IEEE Trans. Power Delivery, Vol. 13, pp. 330-335, 1998.

[14] D. Pommerenke, I. Krage, W. Kalkner, E. Lomke and P. Schmiegel, "On-site PD Measurement on High Voltage Cable Accessories Using Integrated Sensors", Proc. 9th ISH, pp. 1-4, 1995.

[15] E. Lemke, T. Strehl and D. Ru $\beta$ wurm, "New Development in the Field of PD Detection and Location in Power Cables under On-site Condition", Proc. 11th ISH, London, 1999.

[16] P. Craatz, R. Plath, R. Heinrich and W. Kalkner, "Sensitive On-site PD Measurement and Location Using Directional Coupler Sensors in $110 \mathrm{kV}$ Prefabricated Joints", Proc. 11th ISH, Vol. 5, pp. 317-321, London, 1999.

[17] D. Pommerenke, T. Strehl, R. Heinrich, W. Kalkner, F. Schmidt and W. Wei $\beta$ enberg, "Discrimination Between Internal PD and Other Pulses Using Directional Coupling Sensors on HV Cable Systems", IEEE Trans. DEI, Vol. 6, pp. 814-824, 1999.

[18] C. Qiu and N.-Wang, "Techniques for Measuring Partial Discharges in Electrical Apparatus", Mechanical Industry Press, China, 1994 (in Chinese).

[19] CIGRE WG 21-05, "Partial Discharge Detection by Means of Acoustic Detection", 1993.

[20] M. Ekberg, A. Gustafsson, M. Leijon, T. Bengtsson, T... Eriksson, C. Tornkvist, K. Johansson and L. Ming, "Recent Results in HV Measurement Techniques", IEEE Trans. DEI, Vol. 2, pp. 906-913, 1995

[21] L. E. Lundgaard and W. Hansen,-' "Acoustic Method for Quality Control and In-Service Periodic Monitoring of Medium Voltage Cable Terminations", IEEE Intern. Symposium on Electrical Insulation,Virginia, USA, p. . 130-133, 1998.

[22] Y. Tian, P. L. Lewin, A. E: Davies and G. Hathaway, "Acoustic Emission Techniques for Partial Discharge Detection within Ca- 
ble Insulation", Proc. of 8 th Intern. Conf. Dielectric Materials, Measurements and Applications (DMMA), pp. 503-508, Edinburgh, UK, 2000.

[23] D. Pommerenke, R. Jobava and R. Heinrich, "Electromagnetic Finite Differences Time Domain (FDTD) Modelling of Partial Discharge Coupling Applied to High Voltage Cables and Cable Joints", Proc. of 8th DMMA, pp. 497-502, Edinburgh, UK, 2000.

[24] S. Boggs, A. Pathak and P. Walker, "Partial Discharge XXII: High Frequency Attenuation in Shielded Solid Dielectric Power Cable and Implications Thereof for PD Location", IEEE Electrical Insulation Magazine, Vol. 12, No. 1, pp. 9-16, 1996.

[25] R. A. Chipman, "Theory and Problems of Transmission Lines", p. 93, Published by McGraw-Hill Book Company, USA, 1968.
[26] Y. Lu, X. Tan and X. Hu, "PD Detection and Localisation by Acoustic Measurements in an Oil-filled Transformer", IEE Proc.-Sci. Meas. Technol., Vol. 147, pp. 81-85, 2000.

[27] M. Misiti, “Matlab Wavelet Toolbox User's Guide”, Mathworks Company, 1996.

[28] M. Florkowski, "Wavelet Denoising of Partial Discharge Images", Proc. IEEE 6th ICPADM, pp. 459-462, Xi'an, 2000.

[29] X. Ma, C. Zhou and I. J. Kemp, "Investigation Into the Use of Wavelet Theory for Partial Discharge Pulse Extraction in Electrically Noisy Environments", Proc. 8th Intern. Conf. DMMA, pp. 123-126, Edinburgh, UK, 2000. 